\title{
Comparative Studies of Scintillation Properties of Tl-based Crystals
}

\author{
Takayuki Yanagida, ${ }^{*}$ Yutaka Fujimoto, ${ }^{2}$ Miki Arai, ${ }^{2}$ Masanori Koshimizu, ${ }^{2}$ \\ Takumi Kato, ${ }^{1}$ Daisuke Nakauchi, ${ }^{1}$ and Noriaki Kawaguchi ${ }^{1}$ \\ ${ }^{1}$ Nara Institute of Science and Technology, 8916-5 Takayama, Ikoma, Nara 630-0196, Japan \\ ${ }^{2}$ Department of Applied Chemistry, Graduate School of Engineering, Tohoku University, \\ 6-6-07 Aoba, Aramaki, Aoba-ku, Sendai 980-8579, Japan
}

(Received November 16, 2019; accepted February 28, 2020)

Keywords: scintillator, $\mathrm{TlMgCl}_{3}, \mathrm{Tl}_{2} \mathrm{ZrCl}_{6}$, crystal, scintillation detector

Tl-based crystalline scintillators, such as $\mathrm{Tl}_{2} \mathrm{ZrCl}_{6}, \mathrm{TlMgCl}_{3}, \mathrm{TlCdCl}_{3}, \mathrm{TlSrCl}_{3}$, and $\mathrm{TlBaCl}_{3}$, were synthesized by the Bridgeman method. The scintillation light yields of these materials were evaluated under ${ }^{137} \mathrm{Cs} \gamma$-ray irradiation. The relationship between the photoabsorption peak channel and $\gamma$-ray energy was evaluated using various radioisotopes. Furthermore, the same relationship for energy resolution was also investigated.

\section{Introduction}

Phosphor materials have played an important role in radiation detection, ${ }^{(1,2)}$ and they are mainly classified into two types, scintillators and storage phosphors. Scintillators have widespread applications, such as radiation therapy, ${ }^{(3)}$ well logging, ${ }^{(4)}$ environmental monitoring, ${ }^{(5)}$ and astrophysics, ${ }^{(6)}$ particle physics. ${ }^{(7)}$ Storage phosphors have been used for personal dose monitoring and imaging plates. ${ }^{(8-12)}$ Three main types of luminescent material, namely, those with thermally stimulated luminescence (TSL), ${ }^{(13,14)}$ optically stimulated luminescence $(\mathrm{OSL}){ }^{(15,16)}$ and radiophotoluminescence (RPL), ${ }^{(17,18)}$ have long been commonly used as sensor materials.

Recently, halide materials have attracted much attention for scintillator applications since they have a relatively narrower bandgap than oxide and nitride materials, and a narrow bandgap is advantageous for scintillators based on conventional Robbins ${ }^{(19)}$ and some empirical ${ }^{(20,21)}$ models. On the basis of these models, many halide scintillators have been developed, ${ }^{(22-30)}$ and some of them can reach the production stage in some companies. ${ }^{(22-24)}$ Among such halide materials, Tl-based crystalline materials have shown excellent scintillation properties such as high light yield and energy resolution. ${ }^{(31-36)}$ Although the R\&D of scintillators of the emission center doping type is the recent trend, ${ }^{(37-39)}$ these Tl-based scintillators can emit sufficient scintillation photons via intrinsic luminescence. In addition to their efficient scintillation properties, a high effective atomic number owing to $\mathrm{Tl}$ can be expected, and Tl-based scintillators can be potentially attractive for use as $\gamma$-ray scintillation detectors.

*Corresponding author: e-mail: t-yanagida@ms.naist.jp https://doi.org/10.18494/SAM.2020.2711 
In this work, we investigate Tl-based scintillators such as $\mathrm{Tl}_{2} \mathrm{ZrCl}_{6}, \mathrm{TlMgCl}_{3}, \mathrm{TlCdCl}_{3}$, $\mathrm{TlSrCl}_{3}$, and $\mathrm{TlBaCl}_{3}$ focusing on their $\gamma$-ray detection properties. Among them, $\mathrm{Tl}_{2} \mathrm{ZrCl}_{6}{ }^{(33)}$ $\mathrm{TlMgCl}_{3}$, ${ }^{(31)}$ and $\mathrm{TlCdCl}_{3}{ }^{(32)}$ have already been investigated extensively, but $\mathrm{TlSrCl}_{3}$ and $\mathrm{TlBaCl}_{3}$ have not been investigated yet. The aim of this work is to compare the pulse height spectroscopic properties of these newly developed Tl-based scintillators.

\section{Materials and Methods}

Crystal samples of $\mathrm{Tl}_{2} \mathrm{ZrCl}_{6}, \mathrm{TlMgCl}_{3}, \mathrm{TlCdCl}_{3}, \mathrm{TlSrCl}_{3}$, and $\mathrm{TlBaCl}_{3}$ were grown using the vertical Bridgman-Stockbarger technique. The raw materials, in powder form and at least 99.9\% purity, were mixed at a stoichiometric ratio and loaded into precleaned and prebaked quartz ampoules of $8 \mathrm{~mm}$ diameter. The ampoules were dried at $623 \mathrm{~K}$ for $24 \mathrm{~h}$ and sealed in vacuum. The crystal samples were grown using a two-zone furnace at a growth rate of $1.0-2.0 \mathrm{~mm} / \mathrm{h}$ and a temperature gradient of $1.3 \mathrm{~K} / \mathrm{mm}$. After the growth was finished, the ampoules were cooled to room temperature over $24 \mathrm{~h}$. The crystal samples were cut and polished by hand in dry air.

To evaluate $\gamma$-ray detector properties, pulse height spectra were analyzed using our typical setup. The sample was optically coupled with a photomultiplier tube (PMT, R7600-U200, Hamamatsu) using an optical grease (OKEN 6262A). When $\gamma$-rays hit the scintillator, scintillation photons are emitted and converted into photoelectrons by the PMT. After several multiplications, the signal from the PMT was fed into a preamplifier (ORTEC 113), a shaping amplifier (ORTEC 672A) with $6 \mathrm{~ms}$ shaping time, a multichannel analyzer (Amptek Pocket MCA), and finally to a computer. The radioisotopes used in this work were ${ }^{22} \mathrm{Na},{ }^{57} \mathrm{Co},{ }^{109} \mathrm{Cd}$, ${ }^{133} \mathrm{Ba},{ }^{137} \mathrm{Cs},{ }^{152} \mathrm{Eu}$, and ${ }^{241} \mathrm{Am}$, and each photoabsorption peak was approximated by a single Gaussian function to determine the peak channel and energy resolution.

\section{Results and Discussion}

Figure 1 shows the appearance of the sample crystals. All the samples looked white and transparent, and $\mathrm{TlMgCl}_{3}$ and $\mathrm{TlCdCl}_{3}$ in particular showed high transparency to the naked eyes. Although other samples looked opaque in the picture, they actually appeared translucent to the eyes under room light. All the samples had no or low hygroscopicity, so we were able to measure them without any special treatments.

Figure 2 shows pulse height spectra under ${ }^{137} \mathrm{Cs} \gamma$-ray irradiation. Except for $\mathrm{TlBaCl}_{3}$, we detected a clear photoabsorption peak of $662 \mathrm{keV}$. Because $\mathrm{TlMgCl}_{3}$ and $\mathrm{Tl}_{2} \mathrm{ZrCl}_{6}$ showed excellent energy resolution, the escape peak of $\mathrm{Tl}$ was clearly observed, and these results were consistent with pioneering works. ${ }^{(31,33)}$ Table 1 summarizes the scintillation light yield determined by taking into account the quantum efficiency of PMT and energy resolution at $662 \mathrm{keV}$. The light yield of $\mathrm{Tl}_{2} \mathrm{ZrCl}_{6}$ was higher than that reported previously, ${ }^{(33)}$ which could be attributed to the sample condition or crystal quality. Although the energy resolution of $\mathrm{TlMgCl}_{3}$ was worse than that described in a previous report, ${ }^{(31)}$ it would be consistent within a large uncertainty (fitting error). 


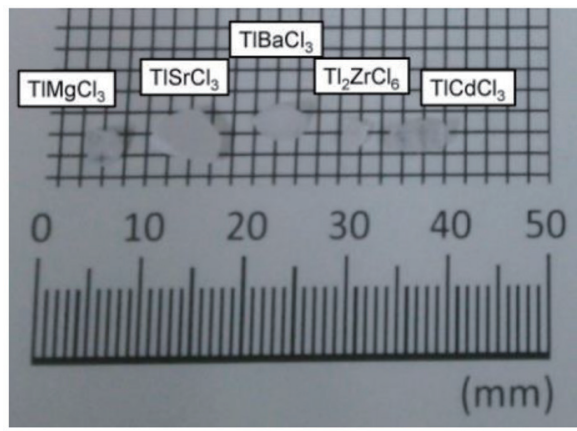

Fig. 1. Samples studied in this work.

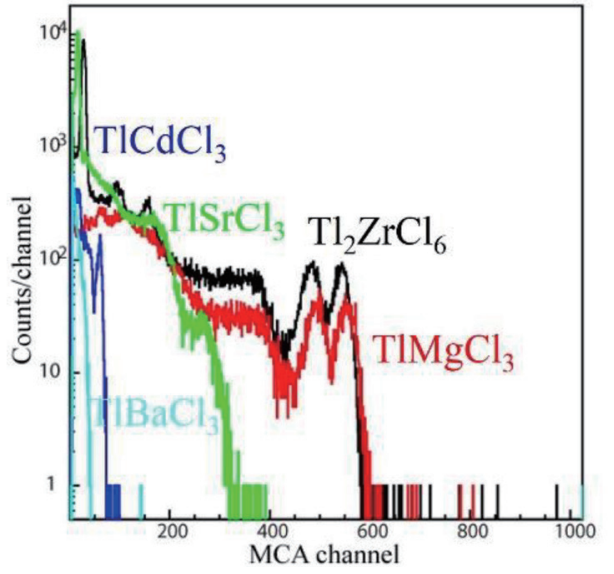

Fig. 2. (Color online) Pulse height spectra of T1based scintillators under $\gamma$-rays from ${ }^{137} \mathrm{Cs}$ excitation.

Table 1

Scintillation light yield ( $\mathrm{ph} / \mathrm{MeV})$ and energy resolution at $662 \mathrm{keV}$ of all the samples.

\begin{tabular}{lcc}
\hline Sample & Light yield (ph/MeV) & Energy resolution at $662 \mathrm{keV} \mathrm{( \% )}$ \\
\hline $\mathrm{Tl}_{2} \mathrm{ZrCl}_{6}$ & $61300 \pm 6100$ & $5.6 \pm 0.2$ \\
$\mathrm{TlMgCl}_{3}$ & $46000 \pm 4600$ & $7 \pm 1.2$ \\
$\mathrm{TlCdCl}_{3}$ & $5900 \pm 600$ & $17 \pm 0.9$ \\
$\mathrm{TlSrCl}_{3}$ & $21300 \pm 2100$ & $10 \pm 2.1$ \\
$\mathrm{TlBaCl}_{3}$ & $<1000$ & - \\
\hline
\end{tabular}

Figure 3 shows the relationship between the photoabsorption peak channel and $\gamma$-ray energy. In this evaluation, the minimum and maximum energies were $22 \mathrm{keV}\left({ }^{109} \mathrm{Cd}\right)$ and $1408 \mathrm{keV}\left({ }^{152} \mathrm{Eu}\right)$, respectively, and we used data points as much as possible if we detected the photoabsorption peak. $\mathrm{TlMgCl}_{3}$ and $\mathrm{Tl}_{2} \mathrm{ZrCl}_{6}$ exhibited the best linear relationship from 22 to $1408 \mathrm{keV}$, and $\mathrm{TlSrCl}_{3}$ showed a linearity from 22 to $662 \mathrm{keV}$. At a higher energy, we did not detect a clear peak in $\mathrm{TlSrCl}_{3}$, possibly owing to the opacity of this sample (Fig. 1). $\mathrm{TlMgCl}_{3}$ and $\mathrm{Tl}_{2} \mathrm{ZrCl}_{6}$ will be applicable for $\gamma$-ray detectors in this energy range.

Figure 4 shows the energy resolution plotted as a function of $\gamma$-ray energy. All the samples showed a monotonic decrease in energy resolution with increasing $\gamma$-ray energy, which was a typical tendency when the fluctuation of photon statistics was a dominant factor for the energy resolution. If we use a photodetector that has a high quantum efficiency at a wavelength longer than $460 \mathrm{~nm}$, a higher energy resolution will be expected for $\mathrm{Tl}_{2} \mathrm{ZrCl}_{6}$ because the quantum efficiency at $460 \mathrm{~nm}$ of the PMT used in this work is $\sim 30 \%$, and photodetectors with high quantum efficiency at longer wavelengths have become available recently. 


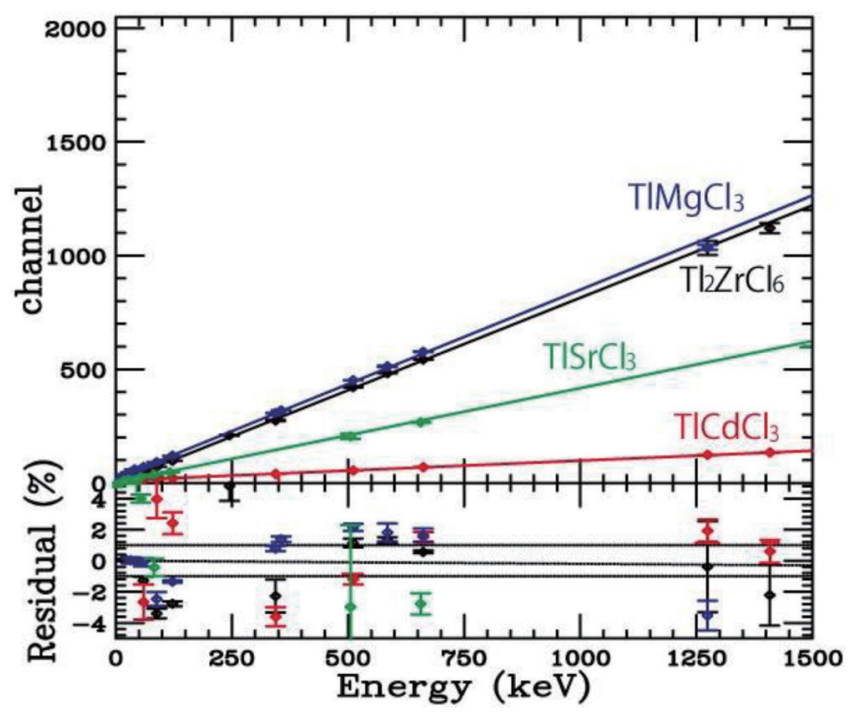

Fig. 3. (Color online) Linearity between peak channel and $\gamma$-ray energy of all samples. The bottom panel shows residuals from linear fitting.

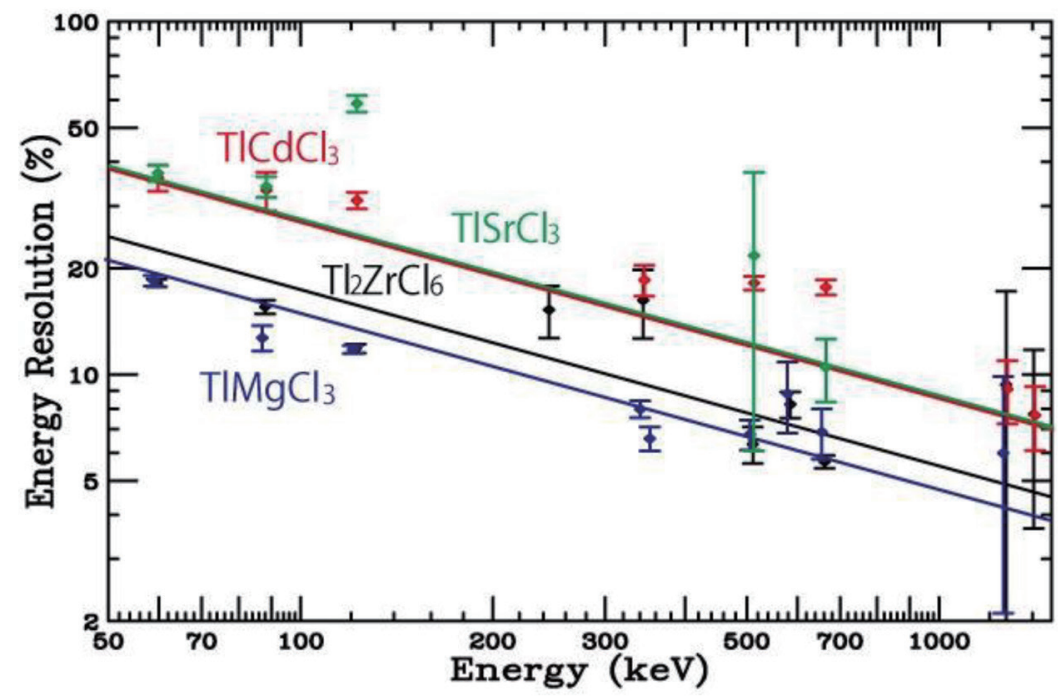

Fig. 4. (Color online) Energy resolution of all samples as function of $\gamma$-ray energy.

\section{Conclusions}

We prepared $\mathrm{Tl}_{2} \mathrm{ZrCl}_{6}, \mathrm{TlMgCl}_{3}, \mathrm{TlCdCl}_{3}, \mathrm{TlSrCl}_{3}$, and $\mathrm{TlBaCl}_{3}$ crystalline scintillators and evaluated their pulse height spectroscopic properties under $\gamma$-ray irradiation. Among these samples, $\mathrm{Tl}_{2} \mathrm{ZrCl}_{6}$ showed the highest light yield of $61300 \pm 6100 \mathrm{ph} / \mathrm{MeV}$ with a good energy resolution of $5.6 \%$ at $662 \mathrm{keV}$. 


\section{Acknowledgments}

This work was supported by Grants-in-Aid for Scientific Research A (17H01375) and B (18H03468 and 19H03533) and Young Scientists B (17K14911). The support from the Cooperative Research Project of the Research Center for Biomedical Engineering, Iketani Foundation, Murata Foundation, and Nippon Sheet Glass Foundation is also acknowledged.

\section{References}

1 T. Yanagida: Proc. Japan Academy, Ser. B, Physical and Biological Sciences 94 (2018) 75.

2 T. Yanagida, G. Okada, and N. Kawaguchi: J. Lumin. 207 (2019) 14.

3 Y. Hirata, K. Watanabe, S. Yoshihashi, A. Uritani, Y. Koba, N. Matsufuji, T. Yanagida, T. Toshito, and K. Fukuda: Sens. Mater. 29 (2017) 1455.

4 N. Kawaguchi, G. Okada, K. Fukuda, and T. Yanagida: Nucl. Instrum. Methods Phys. Res., Sect. A 954 (2019) 161518. https://doi.org/10.1016/j.nima.2018.10.196

5 K. Watanabe, T. Yanagida, K. Fukuda, A. Koike, T. Aoki, and A. Uritani: Sens. Mater. 27 (2015) 269.

6 H. Takahashi, T. Yanagida, D. Kasama, T. Ito, M. Kokubun, K. Makishima, T. Yanagitani, H. Yagi, T. Shigeta, and T. Ito: IEEE Trans. Nucl. Sci. 53 (2006) 2404.

7 T. Ito, T.Yanagida, M. Sato, M. Kokubun, T. Takashima, S. Hirakuri, R. Miyawaki, H. Takahashi, K. Makishima, T. Tanaka, K. Nakazawa, T. Takahashi, and T. Honda: Nucl. Instrum. Methods Phys. Res., Sect. A 579 (2007) 239.

8 E. G. Yukihara and S. W. S. McKeever: Optically Stimulated Luminescence: Fundamentals and Applications (Wiley, 2011).

9 S. W. S. Mckeever: Thermoluminescence of Solids (Cambridge University Press, 1985).

10 J. A. Perry: RPL Dosimetry, Radiophotoluminescence in Health Physics (MEDICAL SCIENCES SERIES) (CRC Press, 1987).

11 J. A. Rowlands: Phys. Med. Biol. 47 (2002) R123.

12 S. W. S. McKeever: Radiat. Meas. 46 (2011) 1336.

13 N. Kawaguchi and T. Yanagida: Sens. Mater. 31 (2019) 1257.

14 N. Kawaguchi, N. Kawano, G. Okada, and T. Yanagida: J. Lumin. 206 (2019) 634.

15 H. Masai, T. Yanagida, G. Okada, A. Koreeda, and T. Ohkubo: Sens. Mater. 29 (2017) 1391.

16 H. Kimura, T. Kato, D. Nakauchi, N. Kawaguchi, and T. Yanagida: J. Mater. Sci.-Mater. Electron. 30 (2019) 16755.

17 G. Okada, K. Shinozaki, D. Shiratori, N. Kawaguchi, and T. Yanagida: Ceram. Int. 45 (2019) 9376.

18 K. Asai, T. Ubukata, M. Koshimizu, Y. Fujimoto, T. Yanagida, H. Kawamoto, and K. Asai: J. Mater. Sci.Mater. Electron. 30 (2019) 10211.

19 D. J. Robbins: J. Electrochem. Soc. 127 (1980) 2694.

20 A. Lempicki, A. Wojtowicz, and A. J. E. Berman: Nucl. Instrum. Methods Phys. Res., Sect. A 333 (1993) 304.

21 P. Dorenbos: Nucl. Instrum. Methods Phys. Res., Sect. A 486 (2002) 208.

22 E. V. D. van Loef, P. Dorenbos, C. W. E. van Eijk, K. W. Kramer, and H. U. Gudel: Nucl. Instrum. Methods Phys. Res., Sect. A 486 (2002) 254.

23 C. M. Combes, P. Dorenbos, C. W. E. van Eijk, K. W. Kramer, and H. U. Gudel: J. Lumin. 82 (1999) 299.

24 T. Yanagida, M. Koshimizu, G. Okada, T. Kojima, J. Osada, and N. Kawaguchi: Opt. Mater. 61 (2016) 119.

25 Y. Fujimoto, K. Saeki, D. Nakauchi, T. Yanagida, M. Koshimizu, and K. Asai: Sens. Mater. 31 (2019) 1241.

26 H. Kimura, T. Kato, D. Nakauchi, M. Koshimizu, N. Kawaguchi, and T. Yanagida: Sens. Mater. 31 (2019) 1265.

27 K. Takahashi, M. Koshimizu, Y. Fujimoto, T. Yanagida, and K. Asai: J. Ceram. Soc. Jpn. 126 (2018) 755.

28 Y. Fujimoto, K. Saeki, D. Nakauchi, H. Fukada, T. Yanagida, M. Koshimizu, and K. Asai: Mater. Res. Bull. 105 (2018) 291.

29 K. Saeki, Y. Fujimoto, M. Koshimizu, D. Nakauchi, H. Tanaka, T. Yanagida, and K. Asai: Jpn. J. Appl. Phys. 57 (2018) 030310.

30 H. Kimura, F. Nakamura, T. Kato, D. Nakauchi, N. Kawano, G. Okada, N. Kawaguchi, and T. Yanagida: Optik 157 (2018) 421.

31 Y. Fujimoto, M. Koshimizu, T. Yanagida, G. Okada, K. Saeki, and K. Asai: Jpn. J. Appl. Phys. 55 (2016) 090301. 
32 Y. Fujimoto, K. Saeki, T. Yanagida, M. Koshimizu, and K. Asai: Radiat. Meas. 106 (2017) 151.

33 Y. Fujimoto, K. Saeki, D. Nakauchi, T. Yanagida, M. Koshimizu, and K. Asai: Sens. Mater. 30 (2018) 1577.

34 R. Hawrami, E. Ariesanti, H. Wei, J. Finkelstein, J. Glodo, and K. S. Shah: J. Cryst. Growth 475 (2017) 216.

35 Q. V. Phan, H. J. Kim, G. Rooh, and S.H. Kim: J. Alloys Compd. 766 (2018) 326.

36 M. Arai, Y. Fujimoto, M. Koshimizu, H. Kimura, T. Yanagida, and K. Asai: Mater. Res. Bull. 20 (2019) 110589.

37 D. Nakauchi, N. Kawaguchi, and T. Yanagida: Sens. Mater. 31 (2019) 1249.

38 H. Fukushima, D. Nakauchi, N. Kawaguchi, and T. Yanagida: Sens. Mater. 31 (2019) 1273.

39 G. Okada, M. Akatsuka, H. Kimura, M. Mori, N. Kawano, N. Kawaguchi, and T. Yanagida: Sens. Mater. 30 (2018) 1547. 